Planetary Systems in the Universe - Observation, Formation and Evolution

Proceedings IAU Symposium No. 202, (C2004 IAU

Alan Penny, Pawel Artymowicz, Anne-Marie Lagrange, 8 Sara Russell, eds.

\title{
Early evolution of the planetary system around PSR B1257+12
}

\author{
Alexander Gusev \\ Kazan University, Dpt. of Gravitation, Kremljevskaja Str., 18, Kazan, \\ 420008, Russia E-mail: Alexander.Gusev@ksu.ru \\ Irina Kitiashvili \\ Kazan University, Dpt. Radioelectronics, Kremljevskaja Str., 18, \\ Kazan, 420008, Russia E-mail: Irina.Kitiashvili@ksu.ru
}

\begin{abstract}
For the plane motion we are completely analyzing the differential equations systems of gravitational capture of the exoplanet at the resonance rotation with action of gravitational and tidal torques by qualitative analysis and bifurcation theory of dynamical systems (DS). The separation of 3-dimensional parameter space of dynamical system by bifurcation surfaces is obtained. The gallery of more than thirty phase portraits of gravitational capture extends the known scenario of cosmogonical evolution of the exoplanet on the early time, when the tidal interaction is very important.
\end{abstract}

It is known the time scale of pulsars is very stable, then in some cases the periodical fluctuation in time of arrival (TOA) may be interpreted as a gravitational perturbation, caused by the planet rotating around the pulsar. Surprise discovery of three planets near the pulsar PSR B1257+12 posed the problems of describing their moving around pulsar, their origin and early rotation.

Demianski and Proszynsky (1979) were first, who established the fact that extra-solar planets is likely to exist: possible mass of the planet around the pulsar PSR $0329+54$ was $0.3 \mathrm{M}_{\oplus}$, orbital radius of 2.3 a.u. and revolution period of 3.3 year. Later, in 1994, Shabanova has informed about existence of another planet around this pulsar, its mass was $2 \mathrm{M}_{\oplus}$ and period of 16.8 years. In 1992 Wolszczan and Frail discovered a planetary system around the pulsar PSR B1257+12 with a period of 6.2 millisecond. The system is the most interesting and "rich" by number of planets $(\mathrm{A}, \mathrm{B}, \mathrm{C})$, it is placed in the Virgo constellation at the distance of $300 \mathrm{pc}$. Two of them B and C move in the resonance 3:2 (Konacki et al. 1999). At present time we know also two multiplanet system around main sequence star (HD 83443, two planets; $\nu$ Andromedae, three planets).

At the early stages of planet evolution the tidal interaction takes a principal role: the tidal effects lead to the capture into resonance rotation of the planet, even if the initial rotation was non-resonance one.

The planet will be considered as a dynamically symmetrical celestial body, when the axis rotation of pulsar is perpendicular to plan orbit of planet. Investigation of the motion equations by qualitative analyses and bifurcation methods permits to obtain the evolution traces at the various values of parameters for extra-solar planets. 


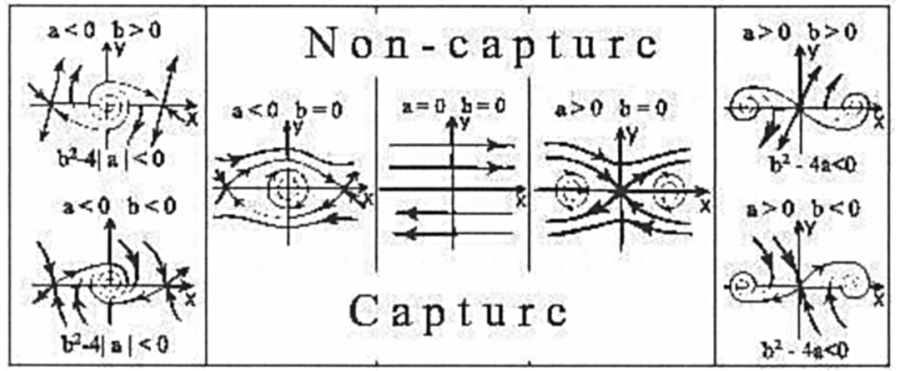

Figure 1. Phase portraits for several values of $a, b, c$

The essence of the orbital evolution calculation can be understand by considering only the principal of conservation of angular momentum. We neglect the inclination of the planet orbit. In according to vector angular momentum theory we can write $\frac{d \vec{L}}{d t}=\vec{M}$, where $\vec{M}$ is the momentum of perturbed forces. Let us to consider the equation system describing the motion nearest to resonance rotation (Beletsky \& Khentov 1995, Salangina 1994):

$$
\dot{x}=y=P(x, y), \dot{y}=a \sin (x)+b y+c=Q(x, y, a, b, c),
$$

where $\mathrm{x}$ is new variable describing a deviation from purely resonance rotation; $a, b, c$ are the parameters of gravitational and tidal interaction. The analyses (Beletsky \& Khentov 1995) is shown that the always resonance is only on ellipse orbit $(e \neq 0)$ except resonance 1:1. This equations have a stable solutions $x=0$ for eccentricity value $e$ less 0.7 . If $e$ more 0.7 , that $x=\frac{\pi}{2}$ is a stable solution. For other resonance $(3: 2,5: 2)$ the resonance motion is observed for $e \neq 0$ only.

The gravitational angular momentum can be provided itself stable resonance rotation, which is described by generalized Cassini lows. However, the initial data of motion can be laid up without a resonance zone. So, should be proposed a mechanism which is provided sufficiently high probability capture at resonance zone. Let us to find a equilibrium states DS $\left(P\left(x_{0}, y_{0}\right)=\right.$ $\left.Q\left(x_{0}, y_{0}, a, b, c\right)=0\right)$ (Gusev \& Salangina 1995) .

As is shown on Fig.1 there are a simple equilibrium state of the saddle, node, focus, center type and/or complicated equilibrium states - saddle-node and the generate saddle-node. To moving we can meet a stable and unstable equilibrium states. The synchronization in DS can been considered as consequence of the strong manifestation of stable, regulated behavior of phase trajectory. Because, a DS should be take a equilibrium states as type a attractive node or focus.

The separation of 3-dimensional parameter space of dynamical systems by bifurcation surfaces and lines we can see on Fig.2. Bifurcation surfaces are: $A=$ $b^{2}-4 \sqrt{a^{2}-c^{2}}=0, B:|a|=|c|$. Bifurcation lines are: coordinate axes $a, b, c$. If $A$ less inside zero, and $|a|$ more than $|c|$ it is unstable node, focus. If $|a|$, $b$ equal zero there are equilibrium state. At the phase space for any parameters are creating or annihilating rough and non-rough dynamical systems of first and second degrees roughness. The conditions $|a|>|c|$ is necessity conditions for existence equilibrium state $x=0$, connecting to resonance rotations of celestial body. 


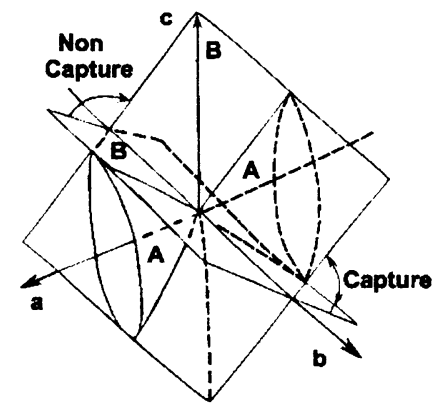

Figure 2. 3-dimensional bifurcation space of parameters

The additional condition $b<0$ is necessary for gravitational capture at resonance rotation of the planet. In this connection the attraction of phase trajectory at resonance zone are flowing qualitative various ways for $c=0$ and $c \neq 0$. For $c=0$ the conditions $b<0(a \neq 0)$ is necessity and sufficient for gravitational capture at resonance zone. For $c \neq 0, b<o$ is an initial data, which is not reduce to capture. We can say that for $c=0$ the probability of capture equal one equal, but for $c \neq 0$ it's lees or equal one. For $b=0$, probability equal zero.

The analyzed DS bifurcations fill total spectrum of the phase portraits reconstruction of gravitational capture, which is typical for the non-rough first and second degrees non-roughness. At the phase space for any parameters rough and non-rough dynamical systems of first and second degrees roughness arise or disappear. Earlier for two-parameter dynamical systems $(a=1, b<0)$ the similar problem has been solved (Neishtadt et al. 1988). Among the bifurcations being investigated we can meet the typical catastrophes of Arnol'd-Thom from $A$ class, which illustrate a structural stability of the gravitational capture phenomenon into the resonance rotation.

\section{References}

Arnold, V.I., Kozlov, V.V., \& Neishtadt, A.I. 1988, in Dynamical systems III, Encyc. of Math. Sci., 3, (New York: Springer)

Beletsky, V.V., \& Khentov, A.A. 1995, Resonance rotation of celestial mechanics (N. Novg.), pp. 430

Demianski, M., \& Proszynsky, M. 1979, Nature, 282, 383

Gusev, A.V., \& Salangina, O.A. 1995 in Int. Conf. Proc. Dynamical systems and chaos, Physics, Vol. 2, ed. Y. Aizawa et al., (Singapore: World Scientific), 418

Konacki, M., Maciejewski, A.J., \& Wolszczan, A. 1999, ApJ, 513, 471

Salangina, O.A., 1994, Master of Science Thesis, Kazan University, Kazan

Shabanova, T.V. 1994, ApJ, 453, 779

Wolszczan, A., \& Frail, D.A. 1992, Nature, 355, 145 